\title{
High-Bandwidth Low-Cost High-Speed Optical Fiber Links using Organic Light Emitting Diodes
}

\author{
P. E. de Souza ${ }^{a}$, N. Bamiedakis ${ }^{a}$, K. Yoshida ${ }^{b}$, P. P. Manousiadis ${ }^{b}$, G. A. Turnbull ${ }^{b}$, I. D. W. Samuel ${ }^{\text {b }}$, \\ R. V. Penty and I. H. White ${ }^{\mathrm{a}}$ \\ ${ }^{a}$ Electrical Engineering Division, Department of Engineering, University of Cambridge, 9 JJ Thomson Avenue, Cambridge, CB3 OFA, UK. \\ ${ }^{b}$ Organic Semiconductor Centre, SUPA, School of Physics and Astronomy, University of St Andrews, St Andrews KY16 9SS, UK. \\ Authore-mail address: ped24@cam.ac.uk
}

\begin{abstract}
Record-high 200 Mbps transmission using an OLED with a $31 \mathrm{MHz} 3 \mathrm{~dB}$ bandwidth using a 3-tap feedforward equaliser is achieved, demonstrating the potential of such devices for use in low-cost polymer optical fiber links.

OCIS codes: (230.3670) Light-emitting diodes; (060.4510) Optical communications.
\end{abstract}

\section{Introduction}

Organic light emitting diodes (OLEDs) hold significant potential for optical interconnects within consumer applications due to their low-cost, relatively straightforward direct deposition manufacturability, and the ability to integrate with flexible substrates and in novel configurations in single process flows. Indeed, OLED displays have seen significant uptake within many products, predominantly due to their low-cost, ease of manufacture and high brightness. Extending this technology to the realms of optical communications has as-yet been underexploited. OLED-based polymer optical fiber and guided-wave links can offer an alternative low-cost method of transmitting information at moderate data rates of a few hundreds of Mbps for use in sensor networks (Internet of Things), inside next-generation cars, airplanes and trains (for an enhanced passenger experience) and within consumer electronics such as in mobile phones and ultra-high definition televisions. Apart from the clear advantages of immunity from electromagnetic interference, reduction in parasitic capacitance effects and impedance matching requirements that optical interconnects exhibit over their electronic counterparts, OLED-based optical links can also be formed on flexible substrates with high-densities and readily integrated with organic electronics. However, current OLED devices have been optimized for display applications and typically exhibit very low bandwidths in the $\mathrm{kHz}$ range that are insufficient to support the necessary data rates. Very little work has been reported on their high-speed performance in optical data links allow current record OLED pulse rates of $100 \mathrm{MHz}$ reported from devices with an area of only $0.01 \mathrm{~mm}^{2}[1]$.

In this work, we present high-bandwidth OLED-based polymer optical fiber links and demonstrate significant improvements in their bandwidth performance through reduction of their size. The smallest device with a $0.12 \mathrm{~mm}^{2}$ area exhibits a $3 \mathrm{~dB}$ bandwidth of $31 \mathrm{MHz}$ and is used to achieve record-high data transmission over a polymer optical fiber link. A net unequalised data rate of $130 \mathrm{Mbps}$ and record $200 \mathrm{Mbps}$ using a simple 3-tap T-spaced feedforward equaliser are achieved using the basic intensity modulation/direct detection (NRZ/OOK) scheme. The results reported here demonstrate the potential of using OLEDs in polymer optical fiber links and indicate that with further optimization of the device structure and the use of advanced modulation formats and equalisation techniques, higher data rates can be achieved for such low-cost optical components.

\section{Organic Light Emitting Diodes}

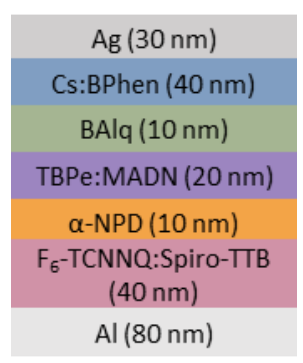

(a)

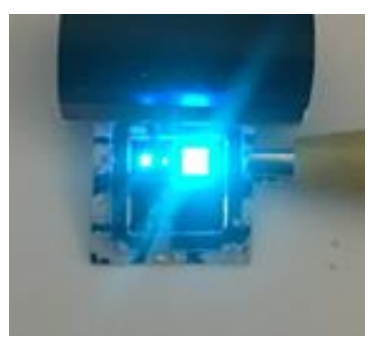

(b)

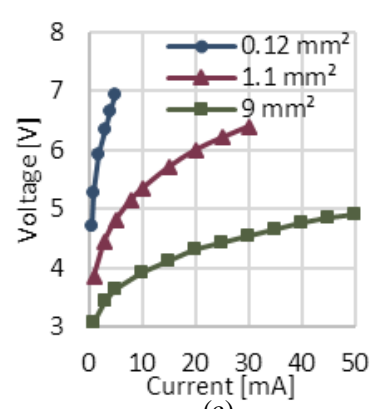

(c)

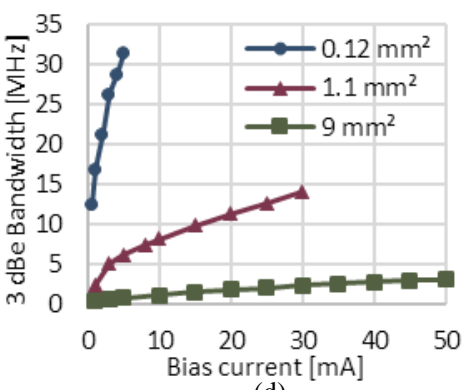

(d)

Fig. 1: (a) The layers of the OLED stack, (b) photograph of the bright blue OLEDs emitting at $495 \mathrm{~nm}$ in three dimensions (1.1, 0.12 and $9 \mathrm{~mm}^{2}$ ), (c) voltage-current curves and (d) $3 \mathrm{~dB}$ bandwidth for the three dimensions of OLEDs. 
The OLEDs presented in this work are $495 \mathrm{~nm}$ top-emitting $0.12 \mathrm{~mm}^{2}, 1.1 \mathrm{~mm}^{2}$ and $9 \mathrm{~mm}^{2}$ small molecule devices with a layer structure as defined in Fig. 1(a). The layers are thermally evaporated in a high vacuum onto $675 \mu \mathrm{m}$ silicon substrates with $300 \mathrm{~nm}$ of $\mathrm{SiO}_{2}$ grown on the surface, before being encapsulated using epoxy and $0.6 \mathrm{~mm}$ glass lids. Fig. 1(c) shows the current- characteristic for the 3 different OLEDs while Fig. 1(d) shows their respective $3 \mathrm{~dB}$ bandwidth. It can be clearly observed that the reduction of the OLED size results in a significant increase in device bandwidth, which can be attributed to the reduced RC component for the small devices. The smallest $0.12 \mathrm{~mm}^{2}$ OLEDs achieve a $31 \mathrm{MHz} 3 \mathrm{~dB}$ bandwidth.

\section{OLED Modulation Bandwidth}

A vector network analyser (Keysight N9913A) and an avalanche photodiode (APD) receiver are used to measure the $S_{11}$ and $S_{21}$ response of the OLEDs. These are used to fit the parameters for a small-signal equivalent circuit (Fig. 2a), extracting the device capacitance and fitting the luminescent proportion $\left(R_{\text {lum }}\right)$ of the total device resistance.

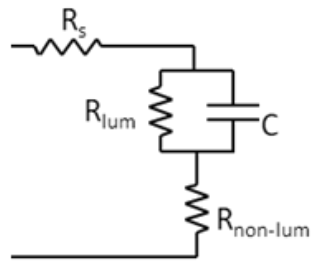

(a)

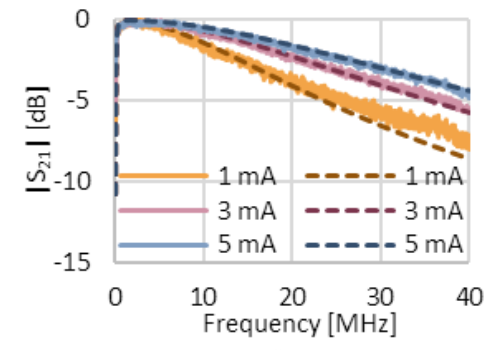

(d)

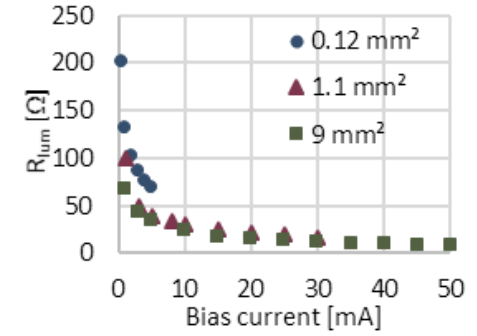

(b)

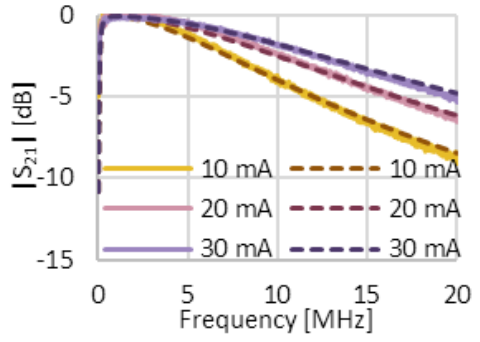

(e)

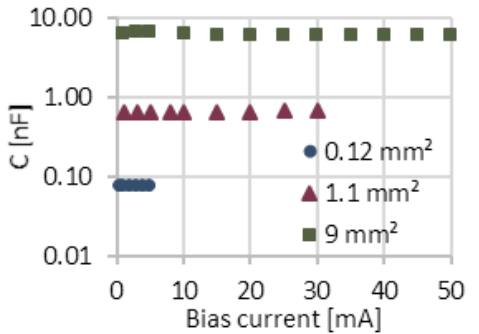

(c)

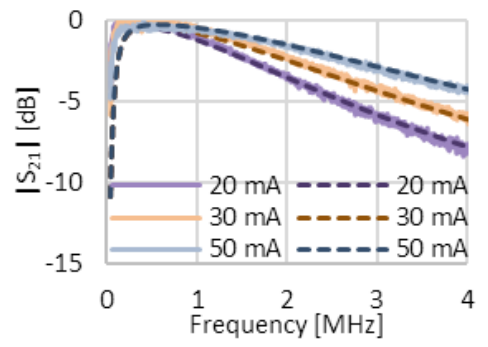

(f)

Fig. 2: (a) The small signal model used to evaluate the $S_{11}$ response, and the resultant (b) luminescent resistances and (c) capacitances at different bias currents; the measured (solid) and fit (dashed) $\mathrm{S}_{21}$ response for (d) the $0.12 \mathrm{~mm}^{2}$, (e) the $1.1 \mathrm{~mm}^{2}$ and (f) the $9 \mathrm{~mm}^{2}$ OLEDs.

The extracted capacitance is found to scale in proportion to area, as shown in Fig. 2(c). The measured $S_{21}$ is used to fit this to a luminescent resistance, $R_{\text {lum }}$, as shown in Fig. 2(b). A good fit is found for the $\mathrm{S}_{21}$ response for all device sizes and bias currents, as shown in Fig. 2(d-f). This approach is valid provided that the exciton lifetime is short compared to the RC time constant. It is suggested that the measured capacitance corresponds to charge accumulation at layer interfaces. From consideration of the recombination current due to drift, the luminescent resistance corresponds to the equivalent resistance for the Langevin recombination rate, $R_{L}$, [2], $R_{\text {lum }}=\frac{E}{A q R_{L}}$, where $E$ is the electric field, $q$ is the electronic charge and $A$ is the device area. The resistance $R_{s}$ and $R_{\text {non-lum }}$ in the equivalent circuit (Fig. 2a) correspond respectively to the device contact resistance and the resistance from the non-luminescent layers within the OLED stack. Simulation studies are under way to further correlate this postulated relationship between $R_{\text {lum }}$ and the semiconductor device parameters and enable the further optimization of the OLED bandwidth performance.

\section{Data transmission experiments}

The setup for the data transmission experiments using the aforementioned $0.12 \mathrm{~mm}^{2}$ OLED is shown in Fig. 3(a). A pulse-pattern generator (PPG, Anritsu MP2010A) modulates the OLED with a $2^{15}-1$ pseudo-random binary sequence and the emitted light is collected by a butt-coupled $1 \mathrm{~mm}$ diameter $(1.5 \mathrm{~m}$ long) plastic optical fiber (POF) patchcord and fed into an avalanche photodiode (APD). The received electrical signal is amplified by two RF amplifiers and filtered by a $180 \mathrm{MHz}$ low-pass filter before being acquired by a digital storage oscilloscope and stored for offline processing. The low data rates employed here do not allow the direct bit-error rate (BER) tests using the BER test set and therefore the BER is estimated using the Q-factor of the recorded eye diagrams [3]. 


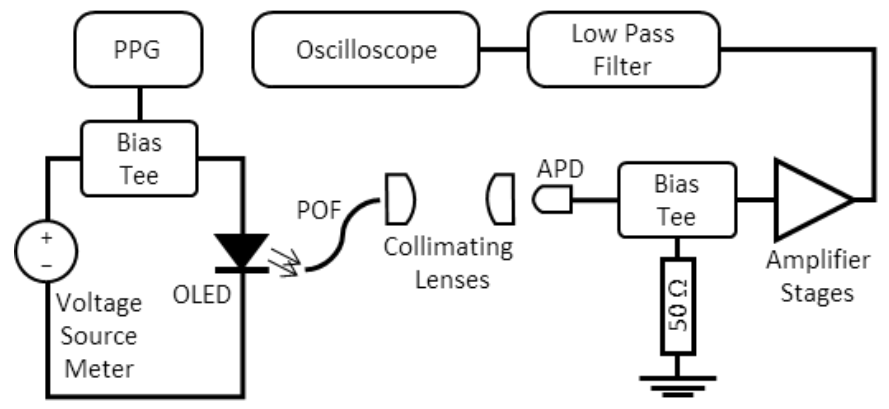

(a)

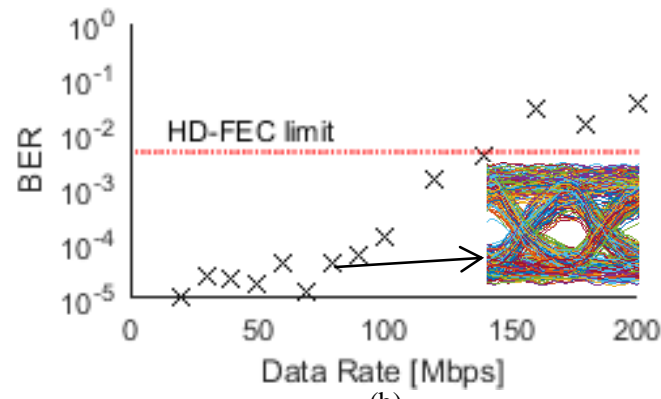

(b)

Fig. 3: (a) Experimental setup for the optical link incorporating the organic LED and (b) the estimated BER at different data rates without equalisation applied. Inset shows the received eye diagram at $80 \mathrm{Mbps}$ transmission.

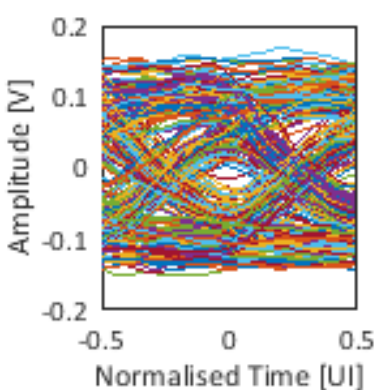

Fig. 4 : Received eye diagrams before and after equalisation at: $140 \mathrm{Mbps}$ (a) and (b), respectively, and $200 \mathrm{Mbps}$ (c) and (d), respectively.

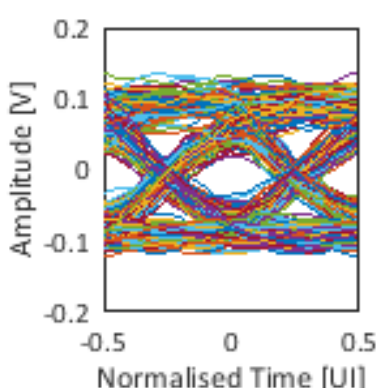

Fig. 4 : Received eye diagrams before and after equalisation at: $140 \mathrm{Mbps}$ (a) and (b), respectively, and $200 \mathrm{Mbps}$ (c) and (d), respectively.

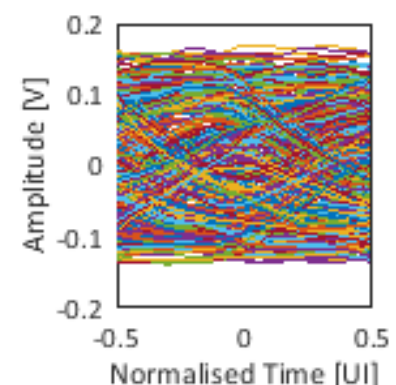

Fig. 4 : Received eye diagrams before and after equalisation at: $140 \mathrm{Mbps}$ (a) and (b), respectively, and $200 \mathrm{Mbps}$ (c) and (d), respectively.

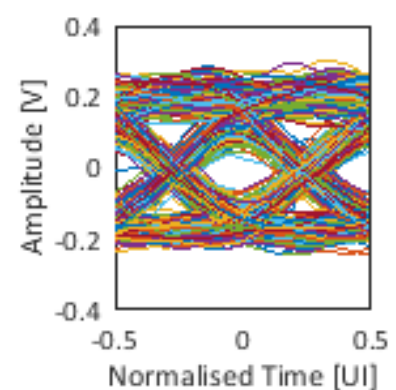

Fig. 4 : Received eye diagrams before and after equalisation at: $140 \mathrm{Mbps}$ (a) and (b), respectively, and $200 \mathrm{Mbps}$ (c) and (d), respectively.

The BER results (Fig. 3(b)) indicate that data transmission up to 140 Mbps with a BER within the hard detection forward error correction (HD-FEC) limit of $4.6 \times 10^{-3}$ can be achieved with these devices [4]. The net unequalised transmission rate is calculated to be $130 \mathrm{Mbps}$ for this link after taking into account the $6.7 \%$ overhead required for the FEC code. The link performance can be improved by applying a simple 3-tap adaptive feedforward equaliser (FFE) at the receiver. Fig. 4 shows the received eye diagrams before and after applying equalisation at the receiver at 140 and 200 Mbps. A clear improvement in eye opening is obtained, while a BER within the FEC limit is achieved for the record $200 \mathrm{Mbps}$ data rate. It is expected that with optimisation of the device structure for high-speed operation and the use of advanced modulation formats and equalisation higher data rates can be achieved from such low-cost links.

\section{Conclusions}

OLEDs are attractive sources for use in low-cost polymer optical fiber and guided-wave links with moderate transmission data rate ( hundreds of Mbps) requirements, such as in sensor networks, within consumer electronics and inside next-generation cars and airplanes. Here, we report high-bandwidth OLED based links using OLEDs with an area of $0.12 \mathrm{~mm}^{2}$ and a $3 \mathrm{~dB}$ bandwidth of $31 \mathrm{MHz}$. Using equalization, we achieve a record-high NRZ data transmission of $200 \mathrm{Mbps}$ over a short polymer optical fiber link. It is hoped that through device optimization and the use of advanced modulation formats that higher data rates towards gigabit-per-second can be achieved using these low-cost components.

\section{Acknowledgements}

This work was supported by the UK EPSRC via the Ultra Parallel Visible Light Communication Project (EP/K00042X/1) and EPSRC Studentship 1466721. Additional data related to this publication is available at the data repository https://doi.org/10.17863/CAM.30619.

\section{References}

[1] H. Kajii, T. Tsukagawa, T. Taneda, and Y. Ohmori, 'Blue organic light-emitting diode as the electro-optical conversion device for highspeed switching applications', J. Phys. D: Appl. Phys., vol. 35, no. 12, p. 1334, 2002.

[2] P. Langevin, 'Recombinaison et mobilities des ions dans les gaz', Annales de Chimie et de Physique, vol. 28, p. 433, 1903.

[3] I. Shake, H. Takara, and S. Kawanishi, 'Simple measurement of eye diagram and BER using high-speed asynchronous sampling', Journal of Lightwave Technology, vol. 22, no. 5, pp. 1296-1302, May 2004.

[4] F. Chang, K. Onohara, and T. Mizuochi, 'Forward error correction for $100 \mathrm{G}$ transport networks', IEEE Communications Magazine, vol. 48, no. 3, pp. S48-S55, Mar. 2010. 